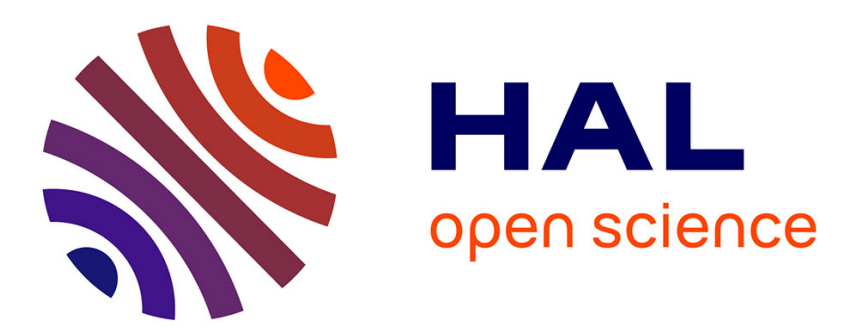

\title{
Transformations structurales dans l'alliage de titane , $\beta$-CEZ études des mécanismes de précipitation après traitement de mise en solution
}

\author{
C. Engelier, J. Béchet
}

\section{- To cite this version:}

C. Engelier, J. Béchet. Transformations structurales dans l'alliage de titane , $\beta$-CEZ études des mécanismes de précipitation après traitement de mise en solution. Journal de Physique IV Proceedings, 1994, 04 (C3), pp.C3-111-C3-116. 10.1051/jp4:1994315 . jpa-00252512

HAL Id: jpa-00252512

https://hal.science/jpa-00252512

Submitted on 1 Jan 1994

HAL is a multi-disciplinary open access archive for the deposit and dissemination of scientific research documents, whether they are published or not. The documents may come from teaching and research institutions in France or abroad, or from public or private research centers.
L'archive ouverte pluridisciplinaire HAL, est destinée au dépôt et à la diffusion de documents scientifiques de niveau recherche, publiés ou non, émanant des établissements d'enseignement et de recherche français ou étrangers, des laboratoires publics ou privés. 


\title{
Transformations structurales dans l'alliage de titane $\beta$-CEZ études des mécanismes de précipitation après traitement de mise en solution
}

\author{
C. ANGELIER et J. BÉCHET
}

\begin{abstract}
Laboratoire de Métallurgie du Conservatoire National des Arts et Métiers, 292 rue Saint Martin, 75141 Paris cedex 03, France
\end{abstract}

\begin{abstract}
The B-CEZ, a high strength titanium alloy developed for acrospace engine applications, is a $\alpha / B$ near $B$ alloy. A wide variety of phase transformations and attendant nodular, lamellar and mixed microstructures are possible according to thermomechanical treatment conditions. The aim of this present paper is to illustrate the influence of solution treatment temperature on equilibrium microstructures and continuous cooling transformations. Solution treatment temperature controls the volume fraction of primary $\alpha$ particles and composition of the $\beta$-matrix. Therefore the transformation during continuous cooling from $\alpha / \mathcal{B}$ or $\mathcal{B}$ field depends on $B$ matrix stability and potential sites amount of $\alpha$ precipitation. After a $B$ solution treatment, the $\alpha$ particles are disappeared and the $B$ phase contains all alloying elements; the continuous cooling transformation produces a Widmanstätten structure. If the cooling rate or/and the solution treatment temperature in the $\alpha / B$ field are sufficiently low, the microstructure consists only of nodular morphology. During $\alpha$ particles growth the $\alpha$ volume fraction increases as equilibrium and the decreasing of growth kinetic leads to supersatured $\beta$ matrix and Widmanstätten $\alpha$ precipitation. The final microstructures are mixed. The influence of solution treat-ment temperature and cooling rate on nucleation and growth mechanisms is specially developed.
\end{abstract}

Les propriétés d'emploi des alliages de titane sont très sensibles à l'histoire thermomécanique qu'ils ont subie. Pour aider au choix approprié des paramètres des gammes de transformations à chaud et des traitements thermiques, il est nécessaire de connaître avec précision les cinétiques des transformations de phase en conditions isothermes et anisothermes. En ce domaine les alliages de titane, et notamment les nuances les plus récentes, sont assez peu documentés.

Nous présentons quelques résultats de l'étude des diagrammes TRC du B-CEZ. Cet alliage de titane à haute résistance (Ti $-5 \% \mathrm{Al}-2 \% \mathrm{Sn}-4 \% \mathrm{Zr}-4 \% \mathrm{Mo}-2 \% \mathrm{Cr}-1 \% \mathrm{Fe}$ ) est destiné à être utilisé notamment en aéronautique [1]. C'est un alliage de type $\alpha / B$ quasi $B$. Pour de tels matériaux, les structures se ramènent souvent à une combinaison de microstructures fines et équiaxes et de microstructures aiguillées selon les gammes de traitements thermomécaniques. Le but de cet article est de montrer l'incidence de la température de mise en solution sur les phénomènes de précipitation se produisant au cours du refroidissement continu qui fait suite. Les aspects morphologiques et cinétiques de la formation de la phase $\alpha$ ont été particulièrement développés. A partir des diagrammes TRC après mise en solution dans le domaine $B$ $\left(920^{\circ} \mathrm{C}\right)$ et dans le domaine $\alpha / B\left(820^{\circ} \mathrm{C}\right.$ ) (figures 1 et $\left.2[2,3]\right)$ nous nous sommes attachés à préciser les rôles respectifs de la germination et de la croissance dans le mécanisme global de la précipitation.

Le traitement de mise en solution contrôle le volume et la composition chimique des deux phases $\alpha$ et $\beta$ présentes à l'équilibre. Ainsi le volume relatif de phase $\alpha$ primaire, équiaxe dans notre cas, diminue au fur et à mesure que la température se rapproche de celle du transus $\left(890^{\circ} \mathrm{C}\right.$ ) (figure 3) selon une expression de la forme:

$$
\% \alpha=67[1-\exp (0,0085(\mathrm{~T}-890))] \text {. }
$$

A l'inverse, la quantité de phase $B$ augmente jusqu'à atteindre $100 \%$ à $890^{\circ} \mathrm{C}$. Sa composition 
Figure 1: Diagramme des transformations en refroidissement continu après mise en solution dans le domaine bêta

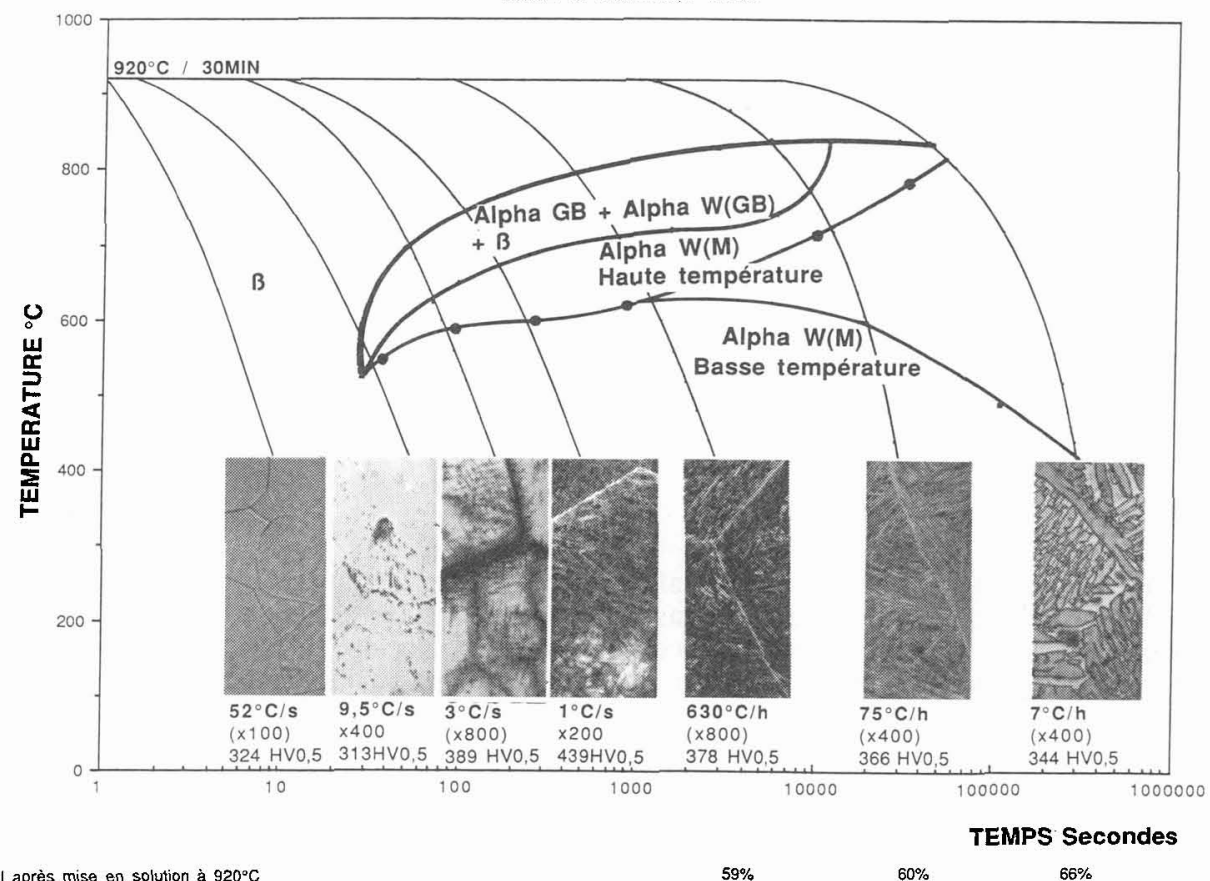

$\%$ Alpha total après mise en solvtion à $920^{\circ} \mathrm{C}$

$59 \%$

$60 \%$

$66 \%$

Figure 2: Diagramme des transformations en refroidissement continu après mise en solution

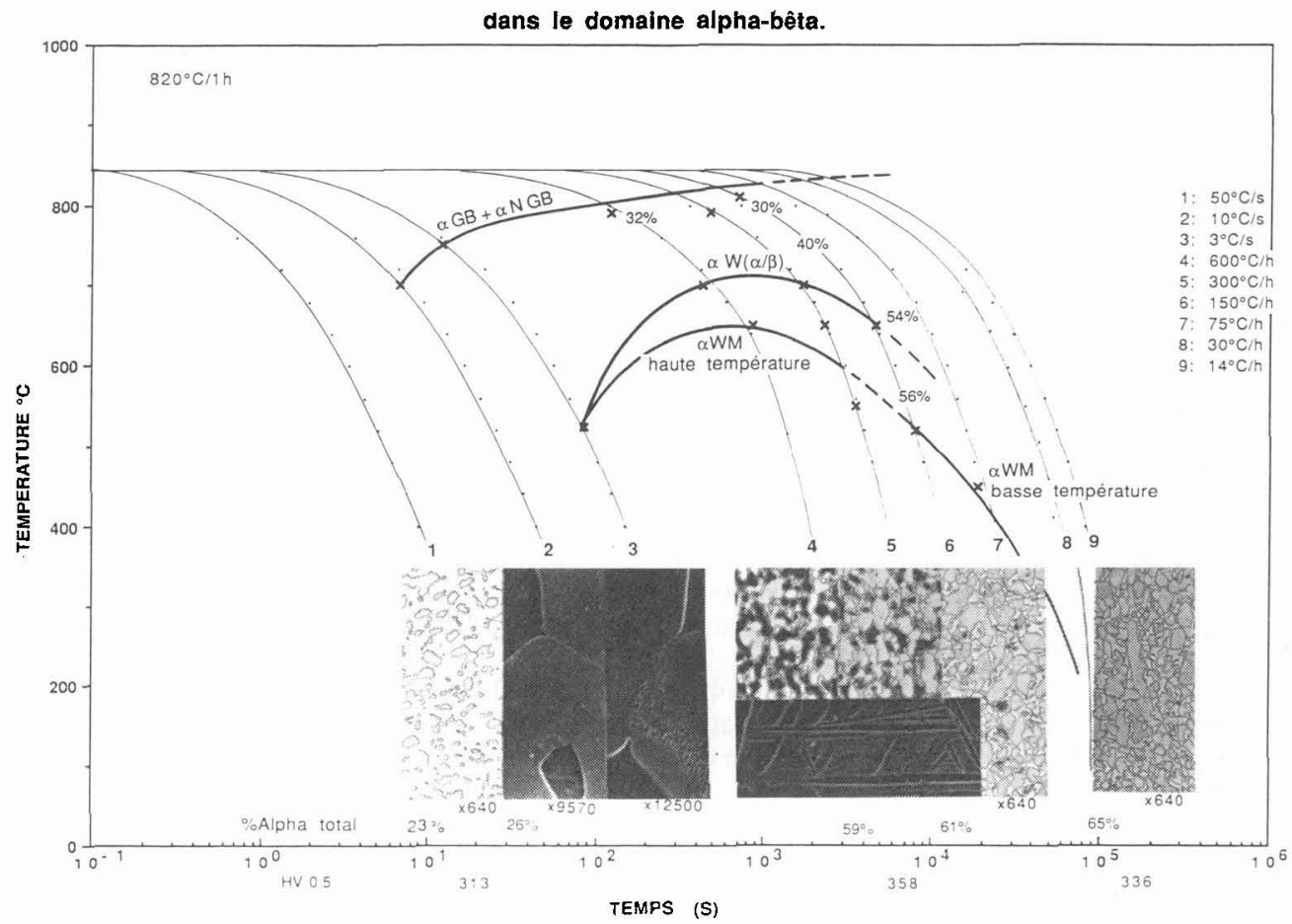


chimique évolue également (figure 4). A $890^{\circ} \mathrm{C}$ la phase $B$ contient tous les éléments d'addition: elle est donc enrichie en élément alphagène (Al) (figure $4 \mathrm{a}$ ) et, par effet de dilution, appauvrie en éléments bêtagènes (Mo, $\mathrm{Cr}$ et $\mathrm{Fe}$ ) (figure $4 \mathrm{~b}$ ). La composition chimique de la phase $\alpha$ reste inchangée quelle que soit la température de mise en solution.

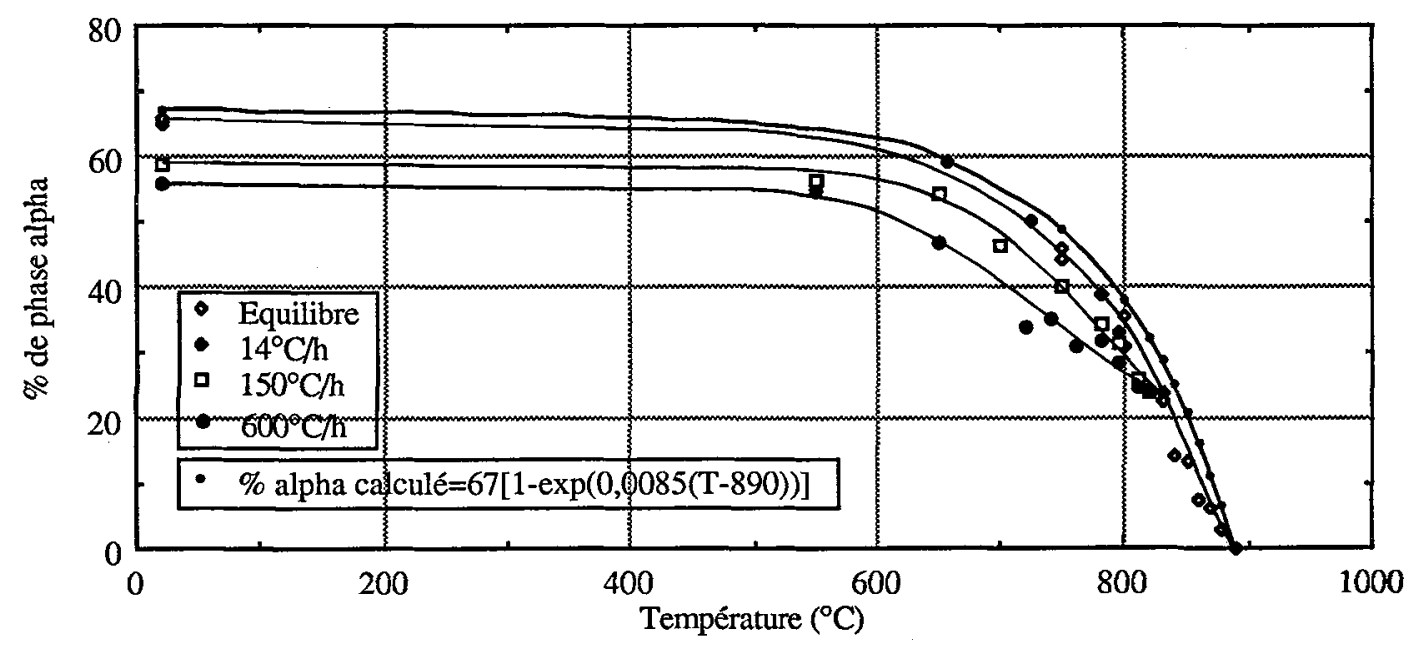

Figure 3: Evolution du volume de phase alpha en fonction de la température de mise en solution et au cours de refroidissements continus depuis $820^{\circ} \mathrm{C}$

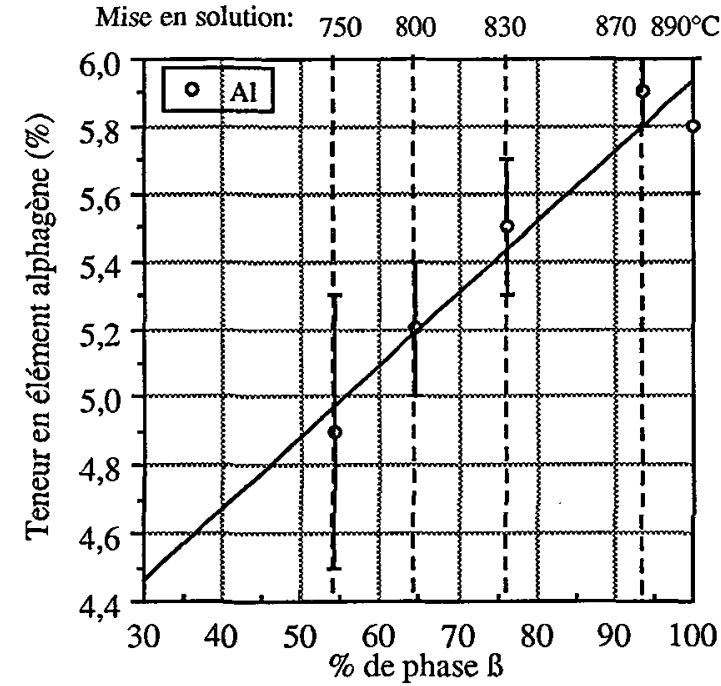

Fig 4a: Teneur en élément alphagène en fonction du volume de phase $B$ à l'équilibre

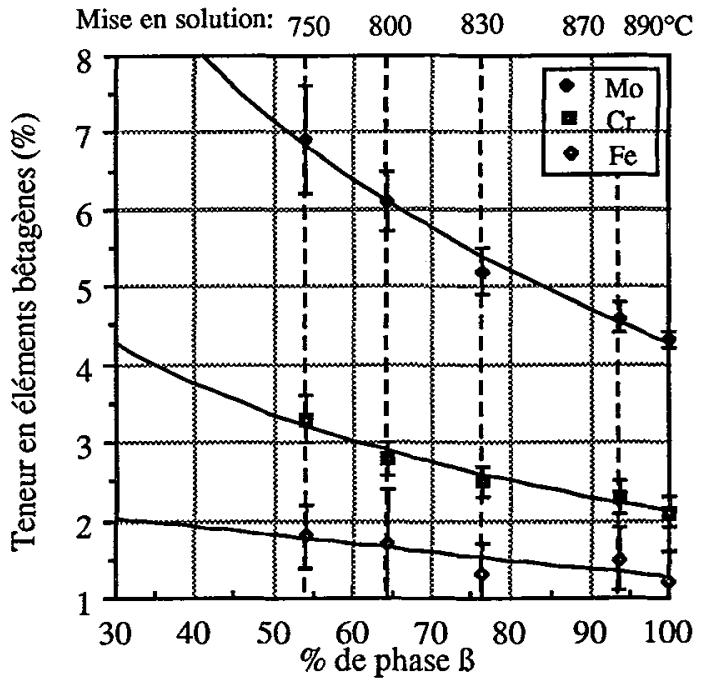

Fig 4b: Teneur en éléments bêtagènes en fonction du volume de phase $\beta$ à l'équilibre

Au cours du refroidissement qui fait suite au traitement de mise en solution dans le domaine B $\left(920^{\circ} \mathrm{C}\right)$, la phase $\beta$ métastable se décompose : il y a précipitation de la phase $\alpha$ et formation d'une structure de type "structure de Widmanstätten", plus ou moins fine selon la vitesse de refroidissement V. La température d'apparition des premiers germes de phase $\alpha$ augmente lorsque $\mathrm{V}$ diminue (figure 1). On peut caractériser la métastabilité de la phase $B$ à cette température par l'écart $\Delta \mathrm{C}$ de composition entre sa teneur 
réelle et la teneur d'équilibre à la température considérée. Nous avons reporté sur la figure 5 la sursaturation $\Delta \mathrm{C}_{\mathrm{Al}}$ de la phase $B$ en aluminium au début de la précipitation en fonction de la vitesse de refroidissement $\mathrm{V}$ depuis $920^{\circ} \mathrm{C}$. La phase $B$ devenant d'autant plus métastable que la température de préci-pitation de la phase $\alpha$ est plus basse, on observe un accroissement de $\Delta C_{A l}$ lorsque $V$ augmente. En effet l'augmentation de la vitesse de refroidissement ayant pour conséquence de réduire les possibilités de diffusion des éléments alphagènes et bêtagènes est associée à une forte sursaturation de la phase $B$, puisque la précipitation ne peut se produire qu'à une température suffisamment basse pour que la taille critique des germes stables soit suffisamment petite. Cependant la sursaturation potentielle est limitée par une valeur maximale correspondant au maintien de la totalité des éléments d'alliage dans la phase $B$ à la température ambiante. On peut donc déterminer assez précisément la vitesse critique de refroidissement qui permet d'éviter toute précipitation et de conserver à $20^{\circ} \mathrm{C}$ la totalité de la phase $B$ métastable: elle est voisine de $40^{\circ} \mathrm{C} / \mathrm{s}$ après mise en solution à $920^{\circ} \mathrm{C}$. Pour les refroidissements les plus lents l'apparition des premiers germes stables de phase $\alpha$ ne peut se faire sans un certain degré de sursaturation. Le minimum nécessaire se situe à environ $0,5 \%$ en masse d'Al. La précipitation de la phase $\alpha$ ne nécessite une plus forte sursaturation que pour des vitesses de refroidissement supérieures à $70^{\circ} \mathrm{C} / \mathrm{h}$.

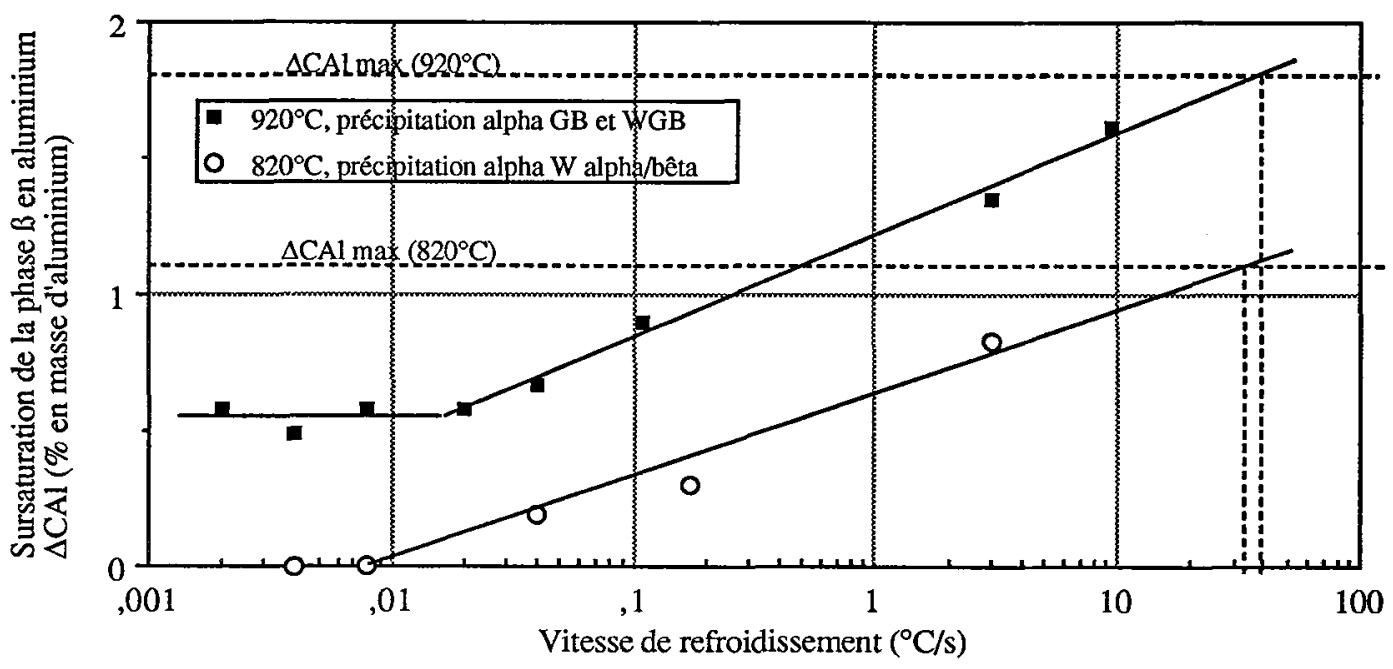

Figure 5: Evolution de la sursaturation en aluminium de la phase $B$ avec la vitesse de refroidissement et la température de mise en solution.

Au cours du refroidissement qui fait suite à la mise en solution dans le domaine $\alpha / B\left(820^{\circ} \mathrm{C}\right)$, la décomposition de la phase $\beta$ peut se produire soit par croissance des nodules de phase $\alpha$ non dissous lors du traitement de mise en solution, soit par précipitation de la phase $\alpha$ aiguillée aboutissant à une structure de Widmanstätten. Dans ce dernier cas, cette précipitation peut être reliée comme précédemment à la métastabilité de la phase $B$, caractérisée par son degré de sursaturation en aluminium $\left(\Delta \mathrm{C}_{\mathrm{Al}}\right)$. La vitesse critique à partir de laquelle la phase $\beta$ est maintenue à la température ambiante est alors de l'ordre de $30^{\circ} \mathrm{C} / \mathrm{s}$. Elle est naturellement inférieure à celle obtenue dans le cas où la mise en solution est réalisée dans le domaine $B$ puisque la sursaturation maximale est nécessairement d'autant plus basse que la phase $B$ contient initialement moins d'aluminium .

Lorque la formation de la phase $\alpha$ ne se produit que par croissance des nodules de phase $\alpha$ primaire le degré de sursaturation de la phase $B$ est nul. La faible vitesse de refroidissement et la présence initiale de la phase $\alpha$ permettent une évolution des volumes précipités tout en conservant la concentration homogène d'équilibre de la matrice $B$. De ce fait, on peut dire que cette croissance est principalement contrôlée par une réaction d'interface. La précipitation aiguillée, correspondant à un $\left(\Delta \mathrm{C}_{\mathrm{Al}}\right)$ non nul, n'intervient que pour les vitesses supérieures à $30^{\circ} \mathrm{C} / \mathrm{h}$.

La cinétique de la décomposition de la phase $B$ au cours du refroidissement depuis la température de mise en solution peut être suivie assez fidèlement par la mesure du volume de phase $\alpha$ formée à toute température intermédiaire. La figure 3 donne un exemple de l'évolution du volume relatif de phase a (mesuré par analyse d'images) en fonction de la température, pour différentes vitesses de refroidissement depuis $820^{\circ} \mathrm{C}$. 
Cependant, dans un souci de compréhension, il peut être intéressant de dissocier les effets propres à chacun des phénomènes mis en jeu et de faire la part respectivement du taux de germination et de la vitesse de croissance sur la cinétique globale de transformation de la phase $\beta$ en phase $\alpha$. On l'a vu en effet ces deux mécanismes sont en compétition selon les conditions de traitement : taux de germination élevé et vitesse de croissance faible lors d'un refroidissement rapide depuis $920^{\circ} \mathrm{C}$; croissance uniquement, par refroidissement lent depuis $820^{\circ} \mathrm{C}$.

Pour les traitements comportant une mise en solution dans le domaine $B\left(a ̀ 920^{\circ} \mathrm{C}\right)$, une estimation simple de la vitesse de croissance peut être obtenue par la mesure de l'épaisseur E $\alpha$ des lamelles (ou plaquettes) de phase $\alpha$. La technique de granulométrie par ouverture permet d'avoir accès rapidement et de façon globale à ce type de paramètre microstructural [4]. La croissance étant directement liée à la mobilité des atomes, elle joue un rôle d'autant plus marqué que la vitesse de refroidissement diminue, comme l'indique la variation de E $\alpha$ en fonction de V (figure 6).

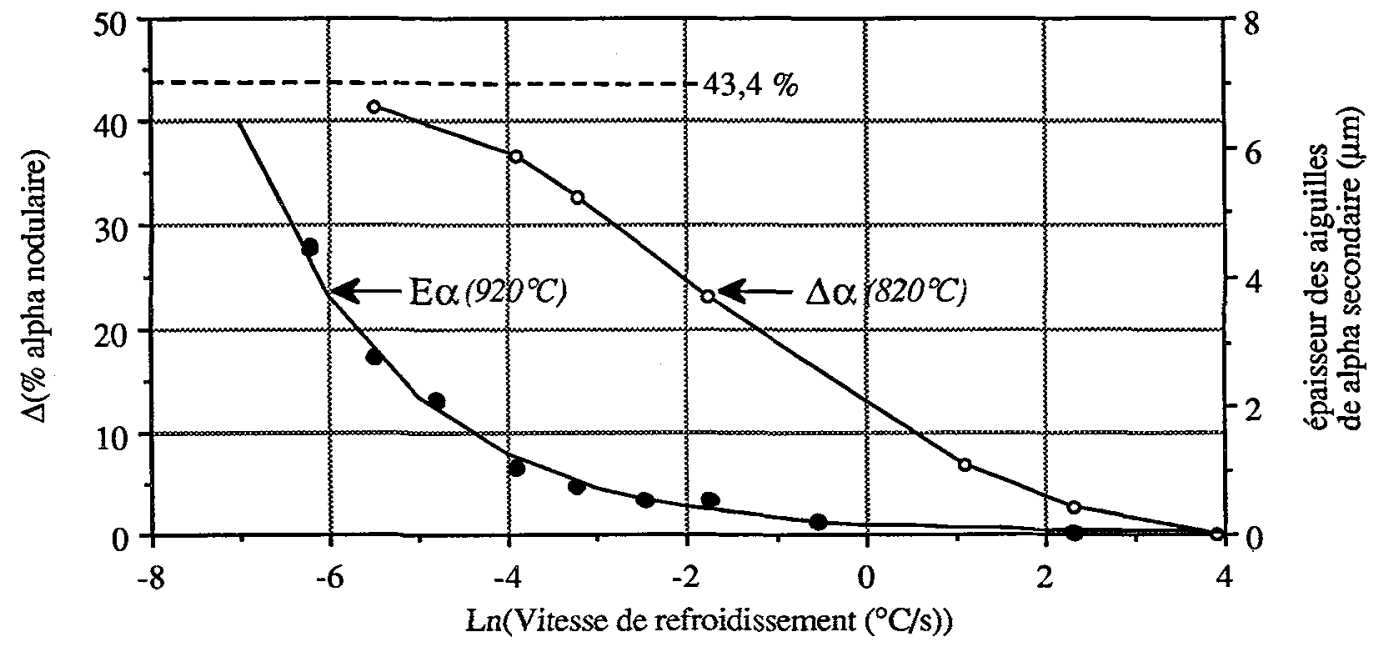

Figure 6: Estimation du taux de croissance en fonction de V (après chauffage à 920 ou $820^{\circ} \mathrm{C}$ ).

D'un autre côté, le taux de germination peut être approché par le rapport $\mathrm{S} / \mathrm{E}^{2}$, où $\mathrm{S}$ est la surface relative occupée par le phase $\alpha$ et E l'épaisseur des lamelles correspondantes. Ce rapport est égal, à un facteur d'échelle près, au nombre de lamelles présentes, c'est-à-dire au nombre de germes apparus, en admettant que leur croissance se fasse de façon homothétique. On voit que lorsque la vitesse de refroidissement diminue au-dessous de la vitesse critique $\mathrm{Vc}$ le nombre de germes apparus augmente à partir de 0 , passe par un maximum pour $\mathrm{V}$ de l'ordre de $3^{\circ} \mathrm{C} / \mathrm{s}$ et ensuite décrôit de façon asymptotique vers 0 , comme le montre la figure 7 . Ce comportement pourrait s'interpréter en termes de dimension critique du germe et de mobilité des atomes, ces deux paramètres évoluant séparément tout au long du refroidissement jusqu'à une température telle qu'il se produit leur conjonction provoquant la germination.

Pour les traitements comportant une mise en solution dans le domaine $\alpha / \mathrm{B}$ (à $820^{\circ} \mathrm{C}$ ), il faut retenir d'autres paramètres microstructuraux. Dans ce cas, la vitesse de croissance peut être représentée par l'accroissement de la quantité de phase $\alpha$ nodulaire $\Delta \alpha$ n obtenue en fin de refroidissement. En fait, pour simplifier, nous rassemblons dans ce paramètre tout ce qui ne relève pas de la fine précipitation intragranulaire aiguillée de phase $\alpha$. La variation de $\Delta \alpha n$ en fonction de $V$ (figure 6 ) est analogue à celle de $\mathrm{E} \alpha$ obtenue dans le cas d'une mise en solution à $920^{\circ} \mathrm{C}$ : la croissance est d'autant plus active que la vitesse de refroidissement est faible. Cependant, après mise en solution à $820^{\circ} \mathrm{C}$, elle s'amorce même pour des valeurs de V élevées : après refroidissement de $10^{\circ} \mathrm{C} / \mathrm{s}$ par exemple, l'accroissement en volume de la quantité de phase $\alpha$ nodulaire est de 2,5\%. A l'opposé, aux faibles vitesses, elle est limitée par la quantité de phase $\alpha$ présente à l'équilibre à température ambiante.

Par ailleurs le taux de germination peut être appréhendé par la quantité de phase $\alpha$ aciculaire \%os qui a précipité au sein de la matrice $\beta$. Sa très grande finesse nous permet de penser qu'en première approximation il y a eu peu ou pas de croissance. Le taux de précipitation intragranulaire peut donc être 
considéré comme représentatif du taux de germination. Comme dans le cas d'une mise en solution à $920^{\circ} \mathrm{C}$, l'évolution de \%os en fonction de $\mathrm{V}$ montre l'existence d'une vitesse pour laquelle la germination est maximale (figure 7). Cette vitesse optimale, moins élevée que précédemment, est voisine de $0,2^{\circ} \mathrm{C} / \mathrm{s} . \mathrm{Ce}$ décalage vers les faibles vitesses du maximum observé traduit bien la plus grande stabilité de la phase $B$ après une mise en solution à $820^{\circ} \mathrm{C}$. Cependant, le phénomène de croissance étant comparativement plus actif dans ce dernier cas (figure 6), la quantité totale de phase $\alpha$ présente à température ambiante après une loi de refroidissement donnée (à condition que la vitesse soit au moins supérieure ou égale à $600^{\circ} \mathrm{C} / \mathrm{h}$ ) reste sensiblement constante pour les deux types de mise en solution. Il n'en reste pas moins que les morphologies obtenues sont très différentes, à volumes relatifs de phase $\alpha$ identiques. L'exemple d'un refroidissement à $150^{\circ} \mathrm{C} / \mathrm{h}$ est significatif : après chauffage à $920^{\circ} \mathrm{C}$ et retour à température ambiante, la microstructure est constituée de $59,7 \%$ de phase $\alpha$ répartie en liseré intergranulaire, colonies de plaquettes et fine précipitation intragranulaire. Après chauffage à $820^{\circ} \mathrm{C}$, on observe $58,9 \%$ de phase $\alpha$ nodulaire et environ $1,5 \%$ d'une fine précipitation intragranulaire. L'impact sur les propriétés d'emploi sera donc très différent dans les deux cas.

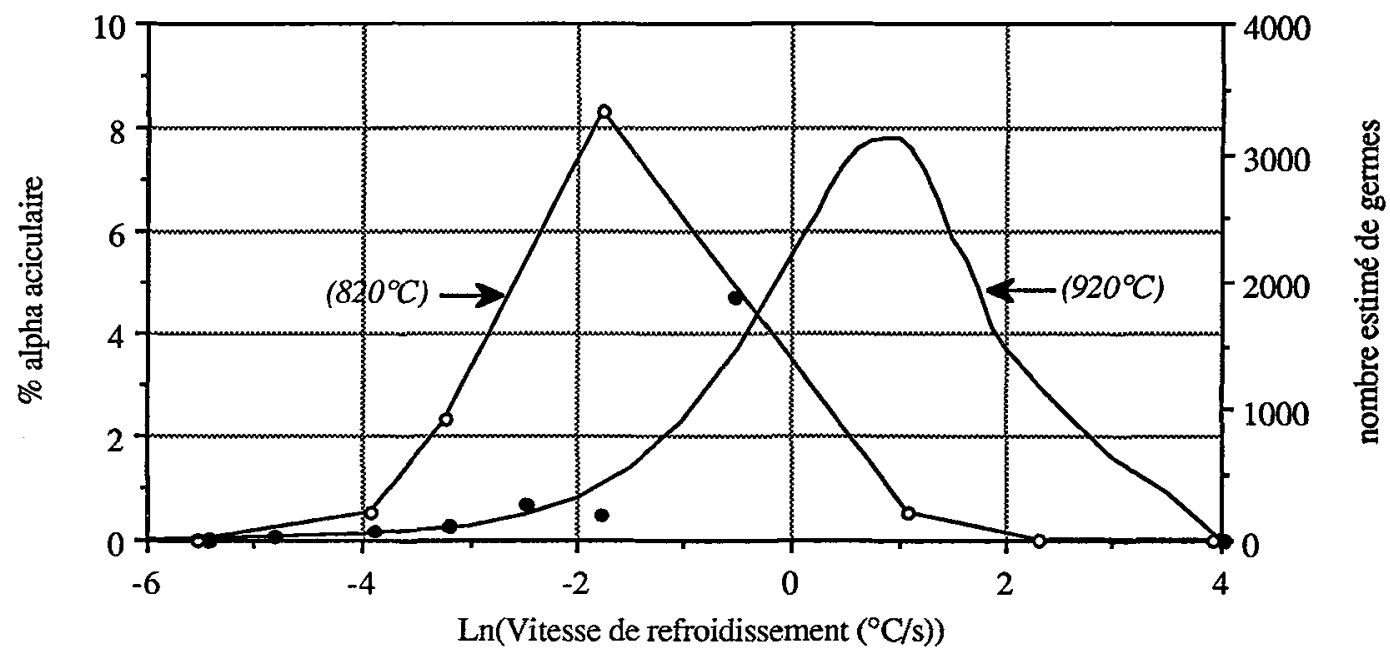

Figure 7: Estimation du taux de germination en fonction de V (après chauffage à 920 ou $820^{\circ} \mathrm{C}$ ).

Il est donc possible de quantifier séparément les phénomènes de germination et croissance intervenant lors de la transformation de la phase $\beta$ en phase $\alpha$. En jouant sur la température de mise en solution et sur la vitesse de refroidissement, on joue sur la métastabilité de la phase $B$, c'est-à-dire sur sa sursaturation potentielle en éléments d'addition. Celle-ci contrôle le déclenchement de la germination d'une structure aiguillée de type Widmanstätten. La présence de phase $\alpha$ primaire non dissoute associée à une plus grande stabilité de la phase $\beta$, favorise la formation de la phase $\alpha$ par croissance. On peut donc intervenir de façon assez précise sur le type de microstructure obtenue après traitement de mise en solution, en modulant la quantité et la morphologie de la phase $\alpha$.

\section{Références}

[1] B. PRANDI, E. ALHERITIERE, F. SCHWARTZ and J. THOMAS - $6^{\circ}$ World Conf. on Ti., Cannes, June 6-9 1988, les Ed. de Physique, Vol.II, pp. 811-818

[2] J. BECHET, C. ANGELIER, B. CORMIER, O. LACAGNE, M. LHERMITTE - Proc.Ti 92, San Diego, à paraître

[3] C. ANGELIER, B. CORMIER et J. BECHET - Colloque GS Titane, Aussois $25-27$ Sept. 1991, CNRS, pp. 217-229

[4] C. ANGELIER, M. POTHRAT, G. FERRIERE et J. BECHET - Colloque GS Titane, Aussois 25-27 Sept. 1991, CNRS, pp. 161-171 